\section{ADHS verzögert das Wachstum}

Forscher untersuchten Größe und Gewicht von 112 Jungen mit ADHS im Alter von 2,4 und 6 Jahren. Als Vergleich dienten 308 gesunde Jungen. In allen Altersgruppen zeigte sich bei ADHS-Kindern ein geringeres Körpergewicht als in der Vergleichsgruppe. Ab der Diagnosestellung kam es allerdings signifikant häufiger zu Übergewicht. Die Körpergröße hingegen war geringer. Die Autoren vermuten, dass niedriges Selbstbewusstsein und soziale Isolation dazu führen, dass die Kinder weniger Sport ausüben und häufiger vor Fernseher und Computer sitzen.

pädiatrie: Kinder-und Jugendmedizin hautnah 2016/1

\section{Folsäure zur Schlag- anfallprävention}

In einer chinesischen Studie wurden 20.702 Patienten mit Hypertonie und ohne bisherigen Schlaganfall untersucht. Sie erhielten entweder Enalapril $10 \mathrm{mg}$ allein oder zusätzlich 0,8 mg Folsäure. In viereinhalb Jahren erlitten $2,7 \%$ der Patienten, die Folsäure und Enalapril und 3,4 \% der Patienten, die nur Enalapril erhielten, einen Schlaganfall. Der Unterschied war signifikant. Auch andere Endpunkte (insbesondere die Kombination aus kardiovaskulärem Tod, Myokardinfarkt und Schlaganfall) unterschieden sich signifikant. Ungeklärt ist, ob die Ergebnisse auf Kaukasier übertragbar sind.

Der Neurologe \& Psychiater 2015/12

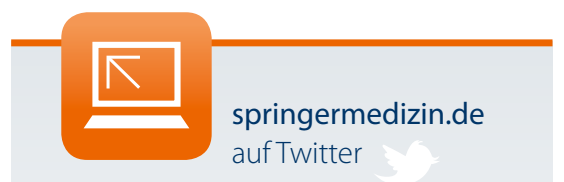

Im Frühling heißt es wieder

"Lauschen" Sie unserem

Zwitschern und werden Sie

zum Follower"

- twitter.com/springermedizin

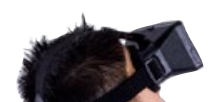 \\ Science Fiction, die funktioniert \\ Virtuelle Räume für Depressive}

Es klingt, als wäre es der Fantasie eines Romanciers entsprungen - doch englische Wissenschaftler wollen in der virtuellen Realität einen Therapieort für Patienten mit Depression gefunden haben.

In einer Studie versetzten die Wissenschaftler 15 depressive Patienten in eine virtuelle Welt. Dazu be-

kamen sie ein Headset, über das ihnen ein dreidimensionales Zimmer vorgespielt wurde. Die Patienten rutschten in die Rolle eines erwachsenen Avatars. Daraufhin spielte das Programm ih- nen ein weinendes kleines Kind vor, das sie mit Worten und Gesten beruhigen sollten. Das Verhalten der Teilnehmer wurde währenddessen aufgezeichnet. Nach einigen Minuten veränderte das Programm die Perspektive. Die Patienten befanden sich nun in der Situation des Kindes und sahen sich dem erwachsenen Avatar gegenüber. Dieser beruhigte sie mit der eigenen, vorher erfassten Stimme. Als die Studienteilnehmer ihr eigenes, mitfühlendes Verhalten wahrnahmen, habe sich das positiv auf ihre Stimmung ausgewirkt. Das hätten drei verschiedene psychologische Tests ergeben. Insgesamt 9 der 15 Patienten berichteten einen Monat nach der Therapie, ihre Depressionen hätten sich deutlich verbessert.

(Anne Bäurle)

\section{Verdacht erhärtet sich}

\section{Blutgruppe beeinflusst Thromboserisiko}

Bereits frühere Studien ließen vermuten, dass Träger der Blutgruppen A, B oder $A B$ stärker gefährdet sind, venöse oder kardiale Thromboembolien zu erleiden. Über die Ausprägung war bisher allerdings wenig bekannt.

Wissenschaftler um Senthil K Vasan, Stockholm, haben deshalb einen Datensatz mit über einer Million gesunder Blutspender aus Schweden und Dänemark ausgewertet und mit den entsprechenden Daten von Patientenregistern in Beziehung gesetzt. In einem Follow-up von 12,5 Jahren fanden sich 9.170 venöse Thromboembolien (VTE) und 24.653 kardiovaskuläre Ereignisse. Für Blutspender mit Blutgruppe $\mathrm{A}, \mathrm{B}$ oder $\mathrm{AB}$ war das relative Risiko für tiefe Venenthrombosen und Lungenembolien im
Vergleich zu 0-Blutgruppenträgern um fast das Doppelte erhöht (InzidenzrateRatio, IRR: 1,92 und 1,80). Ein besonders 0-Blutgruppenträgern wiesen Personen mit Blutgruppe AB auf. Ebenfalls signifikant, aber geringer ausgeprägt, war das Risiko für Nicht-0-Blutgruppenträger, ein kardiales Ereignis wie Herzinfarkt oder Schlaganfall zu erleiden (IRR: 1,10 und 1,07). Nach Ansicht von Vasan und Kollegen könnte es deshalb sinnvoll sein, die Blutgruppe in Thromboserisiko-Assessments bzw. in Prognose-Scores zu berücksichtigen. Immerhin sei die Bestimmung relativ einfach und werde vermutlich nicht von einer Akut-Phase-Reaktion beeinflusst.

(Veronika Schlimpert) hohes Thromboserisiko im Vergleich zu 